\title{
Studies on Novel Zerovalent Iron Decorated Cellulose Nanofibers for Defluoridation
}

\author{
Nalini Sankararamakrishnan, Shruti Mishra \\ Centre for Environmental Science and Engineering, Indian Institute of Technology Kanpur \\ Kanpur U.P. 208016
}

\section{Extended Abstract}

Water is an essential and important component of the universe and it plays a pivotal role in the proper functioning of the earth's ecosystems. In spite of the several water bodies, potable drinking water is not readily available for millions of people around the world. The main cause of this scenario could be associated with rapid industrialization and population growth. Over seven hundred inorganic and organic micropollutants in water have been reported. Some of these micropollutants are highly carcinogenic and toxic while some have very long residence times in the environment and are neither biotransformable nor biodegradable. Several techniques are available to remove these contaminants from potable water and industrial wastewater which include conventional coagulation, chemical precipitation, ion-exchange, reverse osmosis, electrolysis, electrodialysis, and adsorption. Among these, Adsorption, is a cost effective technique that removes both organic and inorganic contaminants from water. The most widely adsorbents for water treatment are activated carbon, activated alumina (1), and biosorbents like cellulose, chitosan (2). It is well known that cellulose is a natural biodegradable polysaccharide is bestowed with very good features to promote diverse applications. This naturally occurring polysaccharide, (C6H10O5)n, has anhydroglucose rings as the repeat unit with a $\beta-1,4$ glucosidic bond. The excellent properties attributed to this polysaccharide such as biodegradability, good stability, intramolecular hydrogen bonding, etc. prompted us to explore its potential for the effective interaction with contaminants (3). Indeed conversion of natural macro fibers to nano fibers would result in increased surface area and increased sorption. The combination of nanotechnology and chemical modification is an alternative approach offering a new technological platform to improve the binding efficiency of biobased adsorbents. As the size of biosorbents is reduced to nanoscale, the huge increase in the specific area is expected to provide an enhanced density of binding sites on the adsorbent Further, doping of nano particles of zerovalent iron, will further improve the sorption capacity of the sorbent towards the contaminants. It is well known that nano zerovalent iron exhibits high metal scavenging properties owing to the reactive nature of iron present in the zerovalent state. Recently, zerovalent iron (ZVI) has been found useful for the rapid adsorption of $\mathrm{As}(\mathrm{III})$ and $\mathrm{As}(\mathrm{V})(4)$ and its reactivity was found to increase in smaller dimensions i.e., nanoscale zero-valent iron (NZVI). However, because of their advanced dispersive properties, these nano adsorbents with small dimensions are difficult to recycle and may lead to loss of the adsorbent and bring about secondary pollution to the environment $(5,6)$. To overcome these difficulties, embedding of nanoparticles in the membranes with high surface area would lead to practical environmental applications.

Thus, in this work the main novelty is to extract cellulosic nano fibers from a green renewable source such as sugarcane bagasse and dope it with zerovalent iron and address defluoridation. Thus to the best of our knowledge this is the first time we are reporting it. The developed adsorbent will be characterized by various techniques such as SEM, TEM, FTIR, XRD, BET and the favorable experimental conditions, isotherm models, mechanism of interaction, and thermodynamics will also be addressed. It was found that adsorption of fluoride was maximum at $\mathrm{pH} \sim 7$ and equilibrium reached within $3.5 \mathrm{~h}$. The rate of adsorption followed pseudo order kinetics and maximum adsorption capacity of the Zerovalent iron loaded cellulose fibers towards fluoride was found to be $20.83 \mathrm{mg} / \mathrm{g}$.

\section{Acknowledgements}

Funding from Department of Science and technology, New Delhi, India (SR/WOS-A/CS-10/2016) is gratefully acknowledged. 


\section{References}

[1] V. K. Gupta, A. Nayak, B. Bhushan, S. Agarwal, Critic Rev. Environ. Sci. Technol, vol. 45, no. 6, pp. 613-668, 2015.

[2] A. Mudhoo, V. K. Garg, S. Wang, "Removal of heavy metals by biosorption," Environmental Chemistry Letters, vol. 10, no. 2, pp. 109-117, 2012.

[3] A. Demirbas, "Heavy metal adsorption onto agro-based waste materials: A review," J. Hazard. Mat., vol. 157, pp. 220-229, 2008.

[4] A. Gupta, M. Yunus, N. Sankararamakrishnan, "Zerovalent iron encapsulated chitosan nanospheres-A novel adsorbent for the removal of total inorganic Arsenic from aqueous systems," Chemosphere., vol. 86, pp. 150-155, 2012.

[5] A. S. K. Hashmi, G. J. Hutchings, "Gold Catalysis,” Angew. Chem. Int. Ed., vol. 45, pp. 7896-7936, 2006.

[6] Y.-E. Miao, R. Wang, D. Chen, Z. Liu, and T. Liu, "Electrospun Self-Standing Membrane of Hierarchical SiO2@yAlOOH (Boehmite) Core/Sheath Fibres for Water Remediation," ACS Appl. Mater. Interfaces., vol. 4, pp. 53535359, 2012. 